International Mathematical Forum, Vol. 8, 2013, no. 25, 1243 - 1251

HIKARI Ltd, www.m-hikari.com

http://dx.doi.org/10.12988/imf.2013.35107

\title{
The Retentivity of Several Kinds of Chaos under Uniformly Conjugation
}

\author{
Tianxiu $\mathbf{L u} u^{1,2}$, Peiyong $\mathbf{Z h u}^{2}$ and Xinxing $\mathbf{W u}^{2}$ \\ (1) Department of Mathematics \\ Sichuan University of Science and Engineering \\ Zigong, P.R. China \\ lubeeltx@163.com (Tianxiu Lu) \\ (2) School of Mathematical Sciences \\ University of Electronic Science and Technology of China \\ Chengdu, P.R. China
}

Copyright (c) 2013 Tianxiu Lu et al. This is an open access article distributed under the Creative Commons Attribution License, which permits unrestricted use, distribution, and reproduction in any medium, provided the original work is properly cited.

\begin{abstract}
This paper is concerned with chaotic of maps on general metric spaces. It is proved that uniformly conjugation preserves AuslanderYorke's chaoticity, dense chaoticity, dense $\delta$-chaoticity, distributional chaoticity, and distributional chaoticity in a sequence.
\end{abstract}

Mathematics Subject Classification: 54H20, 37C15

Keywords: Auslander-Yorke chaos, dense chaos, distributional chaos, uniformly conjugation

\section{Introduction}

The complexity of a topological dynamical system is intensively discussed since the introduction of the term chaos in 1975 by Li and Yorke ${ }^{[12]}$. That is, if a dynamical system $(X, f)$ has an uncountable set $S \subset X$, and $(x, y)$ is a LiYorke pair for $\forall x, y \in S: x \neq y$, then $(X, f)$ is said to be chaotic in the sense of Li-Yorke. While, the definition of chaos in the sense of Li-Yorke is 
inconvenient in engineering applications. In 1989, R. L. Devaney ${ }^{[11]}$ stated a $^{[}$ definition of chaos, known as Devaney chaos today. A map $f$ is said to be chaotic in the sense of Devaney on $X$ if $f$ is transitive on $X$, the set of periodic points of $f$ is dense in $X$ and $f$ has sensitive dependence on initial conditions. Then, in 1992, Banks ${ }^{[6]}$ proved that if $f:(X, d) \rightarrow(X, d)$ is transitive and has dense periodic points then $f$ has sensitive dependence on initial conditions (where $(X, d)$ is a metric space which has no isolated point). This causes that Devaney's chaoticity is preserved under topological conjugation on generally infinite metric space. And in 2007, C. Tian and G. Chen ${ }^{[2]}$ obtained that LiYorke's chaoticity is preserved under (topological) uniformly conjugation on metric spaces.

This paper discussed the retentivity of Auslander-Yorke chaoticity, dense chaoticity (dense $\delta$-chaoticity), distributional chaoticity (distributional chaoticity in a sequence) under uniformly conjugation. The concept of AuslanderYorke's chaos was stated in 1980 by Auslander and Yorke ${ }^{[5]}$. Then dense chaos and dense $\delta$-chaos are defined in 1992 by L. Snoha ${ }^{[7]}$. Distributional chaos was introduced in 1994 by Schweizer and Smital ${ }^{[1]}$. And L. Wang ${ }^{[8]}$ gave the concept of distributional chaos in a sequence in 2007. Other investigation of chaotic see, for example, refs. $[3,4,9,10]$ and some references cited therein.

In this paper, the notation $\mathbb{N}$ denotes the set of natural numbers and $\mathbb{R}^{+}$ denotes the positive real numbers. $B(x, \varepsilon)(\varepsilon>0)$ denotes the $\varepsilon$-neighborhood of $x$ in a space $X$. The closure of a set $A \subset X$ is denoted by $\bar{A}$. Let $f$ : $X \rightarrow X$ be a map on a metric space $(X, d)$ and $x_{0} \in X$. The (positive or forward) orbit of the point $x_{0}$ is denoted by $O_{f}\left(x_{0}\right)=\left\{x_{n}=f^{n}\left(x_{0}\right)\right\}_{n=0}^{\infty}=$ $\left\{x_{0}, f\left(x_{0}\right), f^{2}\left(x_{0}\right), \cdots\right\}$. Other definitions (e.g., metric space, continuous map, period, periodic point) are as usual.

Definition 1.1 Let $h: X \rightarrow Y$ be a map from a metric space $(X, d)$ into a metric space $(Y, \widehat{d})$. The map $h$ is uniformly continuous if for any $\varepsilon>0$, there exists a $\delta>0$ such that for $\forall x_{1}, x_{2} \in X: d\left(x_{1}, x_{2}\right) \leq \delta, \widehat{d}\left(y_{1}, y_{2}\right)=$ $\widehat{d}\left(h\left(x_{1}\right), h\left(x_{2}\right)\right)<\varepsilon$.

Definition 1.2 Let $h: X \rightarrow Y$ be a map from a metric space $(X, d)$ into a metric space $(Y, \widehat{d})$. The map $h$ is said to be a homeomorphism if it is one-toone and onto, and both $h$ and $h^{-1}$ are continuous. In particular, the map $h$ is said to be an uniform homeomorphism if it is one-to-one and onto, and both $h$ and $h^{-1}$ are uniformly continuous.

Definition 1.3 Let $(X, d)$ and $(Y, \widehat{d})$ be two metric spaces, and $f: X \rightarrow X$ and $g: Y \rightarrow Y$ be two maps. Maps $f$ and $g$ are said to be (topologically) conjugate if there exists a homeomorphism $h: X \rightarrow Y$ such that $h \circ f=g \circ h$, where "o" denotes composition of two maps. In particular, if $h$ is an uniform 
homeomorphism and $f$ and $g$ are h-conjugate, $f$ and $g$ are said to be uniformly conjugate.

If $f$ and $g$ are uniformly conjugate, obviously they are conjugate.

\section{Auslander-Yorke chaos}

Since sensitive dependence on initial conditions (sensitivity for short) is rather intuitively a chaotic property, Auslander and Yorke ${ }^{[5]}$ introduced a definition of chaos by associating sensitivity and transitivity.

Definition 2.1 Let $(X, d)$ be a metric space, $f: X \rightarrow X$ be a map. $f$ is said to be Auslander-Yorke chaotic if $f$ is (topological) transitive and $f$ has sensitive dependence on initial conditions. Where, $f$ is (topological) transitive if for arbitrary two nonempty open sets $U, V \subset X$, there exists $m \in \mathbb{Z}$ such that $f^{m}(U) \cap V \neq \phi . f$ has sensitive dependence on initial conditions if there exists a sensitivity constant $\varepsilon>0$ such that for any $x \in X$, any $\delta>0$, one can find $y \in X$ with $d(x, y)<\delta$ and $n \in \mathbb{N}$ such that $d\left(f^{n}(x), f^{n}(y)\right)>\varepsilon$.

Theorem 2.1 Let $(X, d)$ and $(Y, \widehat{d})$ be two metric spaces. $f: X \rightarrow X$ and $g: Y \rightarrow Y$ be two maps, and $h: X \rightarrow Y$ is an uniform homeomorphism. If $f$ and $g$ are h-conjugate, then $f$ is Auslander-Yorke chaotic in $X$ if and only if $g$ is Auslander-Yorke chaotic in $Y$.

Proof Necessity. $f$ is Auslander-Yorke chaotic in $X$, then $f$ is transitive and $f$ has sensitive dependence on initial conditions.

For any $V_{1}, V_{2}$ are two nonempty open sets in $Y$, one has $U_{1}=h^{-1}\left(V_{1}\right), U_{2}=$ $h^{-1}\left(V_{2}\right)$ are two nonempty open sets in $X$.

Since $f$ is transitive on $X$, then there exists $m \in \mathbb{N}$ such that $f^{m}\left(U_{1}\right) \cap U_{2} \neq$ $\phi$. So,

$g^{m}\left(V_{1}\right) \cap V_{2}=\left[g^{m}\left(h\left(U_{1}\right)\right)\right] \cap\left(h\left(U_{2}\right)\right)=\left[h\left(f^{m}\left(U_{1}\right)\right)\right] \cap\left(h\left(U_{2}\right)\right) \supset h\left(f^{m}\left(U_{1}\right) \cap U_{2}\right) \neq \phi$.

Hence, $g$ is transitive on $Y$.

On the other hand, $f$ is sensitivity, i.e. there exists a sensitivity constant $\varepsilon>0$ such that for any $x \in X$, any $\delta>0$, one can find $y \in X: d(x, y)<\delta$ and $n \in \mathbb{N}$ such that $d\left(f^{n}(x), f^{n}(y)\right)>\varepsilon$.

Since $h^{-1}$ is uniformly continuous, then for the above $\varepsilon>0, \forall h\left(x^{*}\right), h\left(y^{*}\right) \in$ $Y$, there exists $\sigma>0$, if $\widehat{d}\left(h\left(x^{*}\right), h\left(y^{*}\right)\right)<\sigma$, then

$$
d\left(h^{-1}\left(h\left(x^{*}\right)\right), h^{-1}\left(h\left(y^{*}\right)\right)\right)=d\left(x^{*}, y^{*}\right)<\varepsilon .
$$

Put $\widehat{\varepsilon}=\sigma . \forall \widehat{x}=h(x) \in Y, \forall \widehat{\delta}>0$, for the above $y \in X: d(x, y)<\delta$, since $h$ is uniformly continuous, i.e. $y$ converges to $x$ implies $h(y)$ converges to $h(x)$, one has $\widehat{d}(\widehat{x}, \widehat{y})<\widehat{\delta}$ (where $\widehat{y}=h(y))$ and

$$
\widehat{d}\left(g^{n}(h(x)), g^{n}(h(y))\right)=\widehat{d}\left(h\left(f^{n}(x)\right), h\left(f^{n}(y)\right)\right)>\widehat{\varepsilon} .
$$


Otherwise it contradicts to $d\left(f^{n}(x), f^{n}(y)\right)>\varepsilon$.

Hence, $g$ is sensitivity. So $g$ is Auslander-Yorke chaos in $Y$.

By the similar argument, the sufficiency is follows immediately.

\section{Dense chaos}

Chaotic in the sense of Li-Yorke means that a system has an uncountable scrambled set $S$ in which arbitrary $(x, y) \in S \times S: x \neq y$ is a Li-Yorke pair. The definition of dense chaos is based on Li-Yorke pairs too. While, different from Li-Yorke chaos, dense chaos described that whether the Li-Yorke pairs are "everywhere" in the space.

Definition 3.1 Let $(X, d)$ is a metric space. A pair of points $\{x, y\} \subset X$ is said to be a Li-Yorke pair if one has simultaneously

$$
\limsup _{n \rightarrow \infty} d\left(f^{n}(x), f^{n}(y)\right)>0 \text { and } \liminf _{n \rightarrow \infty} d\left(f^{n}(x), f^{n}(y)\right)=0 .
$$

Denotes the set of Li-Yorke pairs of $f$ by

$L Y(f)=\left\{(x, y) \in X^{2}: \limsup _{n \rightarrow \infty} d\left(f^{n}(x), f^{n}(y)\right)>0, \liminf _{n \rightarrow \infty} d\left(f^{n}(x), f^{n}(y)\right)=0\right\}$.

And denotes the set of Li-Yorke pairs with modulus $\delta$ by

$L Y(f, \delta)=\left\{(x, y) \in X^{2}: \limsup _{n \rightarrow \infty} d\left(f^{n}(x), f^{n}(y)\right)>\delta, \liminf _{n \rightarrow \infty} d\left(f^{n}(x), f^{n}(y)\right)=0\right\}$.

Definition 3.2 Let $f: X \rightarrow X$ be a map on a metric space $(X, d)$. The map $f$ is said to be densely chaotic if $\overline{L Y(f)}=X \times X$.

Definition 3.3 Let $f: X \rightarrow X$ be a map on a metric space $(X, d)$. The map $f$ is said to be densely $\delta$-chaotic if $\overline{L Y(f, \delta)}=X \times X$.

Theorem 3.1 Let $(X, d)$ and $(Y, \widehat{d})$ be two metric spaces, $f: X \rightarrow X$ and $g: Y \rightarrow Y$ be two maps, and $h: X \rightarrow Y$ is an uniform homeomorphism. If $f$ and $g$ are $h$-conjugate, then $f$ is densely chaotic on $X$ if and only if $g$ is densely chaotic on $Y$.

Proof Necessity (Sufficiency is similar). For every $y=\left(y_{1}, y_{2}\right) \in Y \times Y$, $y_{1} \neq y_{2}$, there exists $x=\left(x_{1}, x_{2}\right) \in X \times X$ with $x_{1} \neq x_{2}$ such that $y_{1}=h\left(x_{1}\right)$, $y_{2}=h\left(x_{2}\right)$.

For every $\varepsilon>0$, because $h$ and $h^{-1}$ are continuous maps, there exists $\varepsilon_{1}>0$ such that $h\left(B\left(x, \varepsilon_{1}\right)\right) \subset B(y, \varepsilon)$.

Since $f$ is densely chaotic on $X$, i.e. $\overline{L Y(f)}=X \times X$, then $B\left(x, \varepsilon_{1}\right) \cap$ $L Y(f) \neq \phi$. Put $x^{*}=\left(x_{1}^{*}, x_{2}^{*}\right) \in B\left(x, \varepsilon_{1}\right) \cap L Y(f)$, then

$$
\limsup _{n \rightarrow \infty} d\left(f^{n}\left(x_{1}^{*}\right), f^{n}\left(x_{2}^{*}\right)\right)>0 \text { and } \liminf _{n \rightarrow \infty} d\left(f^{n}\left(x_{1}^{*}\right), f^{n}\left(x_{2}^{*}\right)\right)=0 \text {. }
$$

Let $y^{*}=\left(h\left(x_{1}^{*}\right), h\left(x_{2}^{*}\right)\right)$, then $y^{*} \in B(y, \varepsilon)$, and 


$$
\limsup _{n \rightarrow \infty} \widehat{d}\left(g^{n}\left(h\left(x_{1}^{*}\right)\right), g^{n}\left(h\left(x_{2}^{*}\right)\right)\right)=\limsup _{n \rightarrow \infty} \widehat{d}\left(h\left(f^{n}\left(x_{1}^{*}\right)\right), h\left(f^{n}\left(x_{2}^{*}\right)\right)\right)>0,
$$

where the inequality holds because $h^{-1}$ is uniformly continuous.

Otherwise, if $\limsup _{n \rightarrow \infty} \widehat{d}\left(h\left(f^{n}\left(x_{1}^{*}\right)\right), h\left(f^{n}\left(x_{2}^{*}\right)\right)\right)=0$, then, the limit of an arbitrary convergent subsequence of sequence $\left\{\widehat{d}\left(h\left(f^{n}\left(x_{1}^{*}\right)\right), h\left(f^{n}\left(x_{2}^{*}\right)\right)\right)\right\}_{n=0}^{\infty}$ is 0 . Let $\left\{\widehat{d}\left(h\left(f^{n_{k}}\left(x_{1}^{*}\right)\right), h\left(f^{n_{k}}\left(x_{2}^{*}\right)\right)\right)\right\}_{k=1}^{\infty}$ is an arbitrary convergent subsequence of the sequence $\left\{\widehat{d}\left(h\left(f^{n}\left(x_{1}^{*}\right)\right), h\left(f^{n}\left(x_{2}^{*}\right)\right)\right)\right\}_{n=0}^{\infty}$, then $\lim _{k \rightarrow \infty} \widehat{d}\left(h\left(f^{n_{k}}\left(x_{1}^{*}\right)\right), h\left(f^{n_{k}}\left(x_{2}^{*}\right)\right)\right)=$ 0 . That is,

$$
\forall \sigma>0, \exists N \in \mathbb{N}, \widehat{d}\left(h\left(f^{n_{k}}\left(x_{1}^{*}\right)\right), h\left(f^{n_{k}}\left(x_{2}^{*}\right)\right)\right)<\sigma \text { for every } k>N .
$$

Since $h^{-1}$ is uniformly continuous, then $d\left(f^{n_{k}}\left(x_{1}^{*}\right), f^{n_{k}}\left(x_{2}^{*}\right)\right)<\sigma_{1}$ for every $\sigma_{1}>0$. So the limit of an arbitrary convergent subsequence of sequence $\left\{\widehat{d}\left(f^{n}\left(x_{1}^{*}\right), f^{n}\left(x_{2}^{*}\right)\right)\right\}_{n=0}^{\infty}$ is 0 . Therefore, $\limsup _{n \rightarrow \infty} d\left(f^{n}\left(x_{1}^{*}\right), f^{n}\left(x_{2}^{*}\right)\right)=0$. This is a contradiction.

Similarly, since $h$ is uniformly continuous, one has

$$
\liminf _{n \rightarrow \infty} \widehat{d}\left(g^{n}\left(h\left(x_{1}^{*}\right)\right), g^{n}\left(h\left(x_{2}^{*}\right)\right)\right)=\liminf _{n \rightarrow \infty} \widehat{d}\left(h\left(f^{n}\left(x_{1}^{*}\right)\right), h\left(f^{n}\left(x_{2}^{*}\right)\right)\right)=0 .
$$

So, $y^{*} \in B(y, \varepsilon) \cap L Y(g) \neq \phi$. This implies that $\overline{L Y(g)}=Y \times Y$.

Finally, $g$ thus is densely chaotic on $Y$.

Similarly to the proof of Theorem 4.1, we have

Theorem 3.2 Let $(X, d)$ and $(Y, \widehat{d})$ be two metric spaces, $f: X \rightarrow X$ and $g: Y \rightarrow Y$ be two maps, and $h: X \rightarrow Y$ is an uniform homeomorphism. If $f$ and $g$ are $h$-conjugate, then $f$ is densely $\delta$-chaotic on $X$ if and only if $g$ is densely $\delta$-chaotic on $Y$.

\section{Distributional chaos}

Let $(X, d)$ be a metric space, $f: X \rightarrow X$ be a map, $x, y \in X, t \in \mathbb{R}^{+}, n \in \mathbb{N}$, the upper and lower (distance) distribution functions $F_{x y}^{*}(t, f)$ and $F_{x y}(t, f)$ are defined as follows:

$$
\begin{aligned}
& F_{x y}^{*}(t, f)=\limsup _{n \rightarrow \infty} \frac{1}{n} \sum_{i=1}^{n} \chi_{[0, t)}\left(d\left(f^{i}(x), f^{i}(y)\right)\right) \\
& F_{x y}(t, f)=\liminf _{n \rightarrow \infty} \frac{1}{n} \sum_{i=1}^{n} \chi_{[0, t)}\left(d\left(f^{i}(x), f^{i}(y)\right)\right) .
\end{aligned}
$$

where $\chi_{[0, t)}$ is the characteristic function of the set $[0, t)$, i.e. $\chi_{[0, t)}(s)=1$ when $s \in[0, t)$ or $\chi_{[0, t)}(s)=0$ when $s \notin[0, t)$. 
Definition 4.1 Dynamical system $(X, f)$ is said to be distributional chaotic if there exists an uncountable set $S \subset X$ such that

(1) $\forall t>0, \forall x, y \in S: x \neq y, F_{x y}^{*}(t, f)=1$;

(2) $\exists t>0, \forall x, y \in S: x \neq y, F_{x y}(t, f)=0$.

We called $S$ be a distributionally scrambled set of $X$.

Suppose $\left\{p_{k}\right\}_{k \in \mathbb{N}}$ be a strictly increasing sequence of positive integers. $x, y \in X, t \in \mathbb{R}^{+}$, the upper and lower (distance) distribution functions $F_{x y}^{*}\left(t,\left\{p_{k}\right\}_{k \in \mathbb{N}}, f\right)$ and $F_{x y}\left(t,\left\{p_{k}\right\}_{k \in \mathbb{N}}, f\right)$ are defined as follows:

$$
\begin{aligned}
& F_{x y}^{*}\left(t,\left\{p_{k}\right\}_{k \in \mathbb{N}}, f\right)=\limsup _{n \rightarrow \infty} \frac{1}{n} \sum_{k=1}^{n} \chi_{[0, t)}\left(d\left(f^{p_{k}}(x), f^{p_{k}}(y)\right)\right) \\
& F_{x y}\left(t,\left\{p_{k}\right\}_{k \in \mathbb{N}}, f\right)=\liminf _{n \rightarrow \infty} \frac{1}{n} \sum_{k=1}^{n} \chi_{[0, t)}\left(d\left(f^{p_{k}}(x), f^{p_{k}}(y)\right)\right) .
\end{aligned}
$$

Definition 4.2 Dynamical system $(X, f)$ is said to be distributional chaotic in a sequence if there exists an uncountable set $S \subset X$ such that

(1) $\forall t>0, \forall x, y \in S: x \neq y, F_{x y}^{*}\left(t,\left\{p_{k}\right\}_{k \in \mathbb{N}}, f\right)=1$;

(2) $\exists t_{0}>0, \forall x, y \in S: x \neq y, F_{x y}\left(t,\left\{p_{k}\right\}_{k \in \mathbb{N}}, f\right)=0$.

Theorem 4.1 Let $(X, d)$ and $(Y, \widehat{d})$ be two metric spaces. $f: X \rightarrow X$ and $g: Y \rightarrow Y$ be two maps, and $h: X \rightarrow Y$ is an uniform homeomorphism. If $f$ and $g$ are $h$-conjugate, then dynamical system $(X, f)$ is distributional chaotic if and only if dynamical system $(Y, g)$ is distributional chaotic.

Proof Necessity. $f$ be distributional chaotic, then there exists an uncountable set $S \subset X$ such that

(1) $\forall t>0, \forall x, y \in S: x \neq y, F_{x y}^{*}(t, f)=1$;

(2) $\exists t_{0}>0, \forall x, y \in S: x \neq y, F_{x y}\left(t_{0}, f\right)=0$.

Since $S$ is uncountable and $h$ is one-to-one, then $h(S) \subset Y$ and $h(S)$ is uncountable.

For $\forall t>0, \forall h(x), h(y) \in h(S): h(x) \neq h(y)$, one has $x, y \in S, x \neq y$, and

$$
\begin{gathered}
F_{h(x) h(y)}^{*}(t, g)=\limsup _{n \rightarrow \infty} \frac{1}{n} \sum_{i=1}^{n} \chi_{[0, t)}\left(\widehat{d}\left(g^{i}(h(x)), g^{i}(h(y))\right)\right) \\
=\limsup _{n \rightarrow \infty} \frac{1}{n} \sum_{i=1}^{n} \chi_{[0, t)}\left(\widehat{d}\left(h\left(f^{i}(x)\right), h\left(f^{i}(y)\right)\right)\right)
\end{gathered}
$$


Since $h$ is uniformly continuous, then for the above $t>0, \exists \delta(t)>0$, if some $i \in\{1,2, \cdots, n\}$ satisfied $d\left(f^{i}(x), f^{i}(y)\right)<\delta(t)$, then $\widehat{d}\left(h\left(f^{i}(x)\right), h\left(f^{i}(y)\right)\right)<t$. So

$$
\frac{1}{n} \sum_{i=1}^{n} \chi_{[0, t)}\left(\widehat{d}\left(h\left(f^{i}(x)\right), h\left(f^{i}(y)\right)\right)\right) \geq \frac{1}{n} \sum_{i=1}^{n} \chi_{[0, \delta(t))}\left(d\left(f^{i}(x), f^{i}(y)\right)\right) .
$$

Combined with condition (1), one has

$$
\limsup _{n \rightarrow \infty} \frac{1}{n} \sum_{i=1}^{n} \chi_{[0, t)}\left(\widehat{d}\left(h\left(f^{i}(x)\right), h\left(f^{i}(y)\right)\right)\right) \geq \limsup _{n \rightarrow \infty} \frac{1}{n} \sum_{i=1}^{n} \chi_{[0, \delta(t))}\left(d\left(f^{i}(x), f^{i}(y)\right)\right)=1 .
$$

And because

$$
\limsup _{n \rightarrow \infty} \frac{1}{n} \sum_{i=1}^{n} \chi_{[0, t)}\left(\widehat{d}\left(h\left(f^{i}(x)\right), h\left(f^{i}(y)\right)\right)\right) \leq 1
$$

it followed that

$$
F_{h(x) h(y)}^{*}(t, g)=\limsup _{n \rightarrow \infty} \frac{1}{n} \sum_{i=1}^{n} \chi_{[0, t)}\left(\widehat{d}\left(h\left(f^{i}(x)\right), h\left(f^{i}(y)\right)\right)\right)=1 .
$$

On the other hand, by condition (2), there exists $t_{0}>0$ such that for any $x, y \in S: x \neq y$,

$$
F_{x y}\left(t_{0}, f\right)=\liminf _{n \rightarrow \infty} \frac{1}{n} \sum_{i=1}^{n} \chi_{\left[0, t_{0}\right)}\left(d\left(f^{i}(x), f^{i}(y)\right)\right)=0 .
$$

Since $h^{-1}$ is uniformly continuous, there exists $\delta\left(t_{0}\right)>0$, if some $i \in\{1,2, \cdots, n\}$ satisfied

$$
\widehat{d}\left(h\left(f^{i}(x)\right), h\left(g^{i}(y)\right)\right)<\delta\left(t_{0}\right)(i \in\{1,2, \cdots, n\}),
$$

then $d\left(f^{i}(x), f^{i}(y)\right)<t_{0}$.

This implies that,

$$
\begin{aligned}
F_{h(x) h(y)}\left(\delta\left(t_{0}\right), g\right) & =\liminf _{n \rightarrow \infty} \frac{1}{n} \sum_{i=1}^{n} \chi_{\left[0, \delta\left(t_{0}\right)\right)}\left(\widehat{d}\left(g^{i}(h(x)), g^{i}(h(y))\right)\right) \\
& =\liminf _{n \rightarrow \infty} \frac{1}{n} \sum_{i=1}^{n} \chi_{\left[0, \delta\left(t_{0}\right)\right)}\left(\widehat{d}\left(h\left(f^{i}(x)\right), h\left(f^{i}(y)\right)\right)\right) \\
& \leq \liminf _{n \rightarrow \infty} \frac{1}{n} \sum_{i=1}^{n} \chi_{\left[0, t_{0}\right)}\left(d\left(f^{i}(x), f^{i}(y)\right)\right)=0 .
\end{aligned}
$$


Meanwhile, noting that

$$
\liminf _{n \rightarrow \infty} \frac{1}{n} \sum_{i=1}^{n} \chi_{\left[0, \delta\left(t_{0}\right)\right)}\left(\widehat{d}\left(g^{i}(h(x)), g^{i}(h(y))\right)\right) \geq 0
$$

clearly,

$$
F_{h(x) h(y)}\left(\delta\left(t_{0}\right), g\right)=\liminf _{n \rightarrow \infty} \frac{1}{n} \sum_{i=1}^{n} \chi_{\left[0, \delta\left(t_{0}\right)\right)}\left(\widehat{d}\left(g^{i}(h(x)), g^{i}(h(y))\right)\right)=0
$$

Therefore, $h(S)$ is an uncountable distributionally scrambled set of $Y$. We thus conclude that $(Y, g)$ is distributional chaotic.

Similarly to the discussion of Theorem 4.1, Theorem 4.2 is obtained.

Theorem 4.2 Let $(X, d)$ and $(Y, \widehat{d})$ be two metric spaces. $f: X \rightarrow X$ and $g: Y \rightarrow Y$ be two maps, and $h: X \rightarrow Y$ is an uniform homeomorphism. If $f$ and $g$ are $h$-conjugate, then dynamical system $(X, f)$ is distributional chaotic in a sequence if and only if dynamical system $(Y, g)$ is distributional chaotic in a sequence.

Remark 1. It is easy to check that chaos are preserved under uniformly conjugation if they are preserved under topological conjugation.

Remark 2. There are some problems for research. For example, topological conjugation preserves Li-Yorke's chaoticity (Auslander-Yorke chaoticity, dense chaoticity, dense $\delta$-chaoticity, distributional chaoticity and distributional chaoticity in a sequence) or not? Can the chaos be extended to topological spaces? If they can be, topological conjugation (or uniformly conjugation) preserves them or not?

\section{Acknowledgments}

We would like to express our thanks to the experts for their valuable suggestions. This work was supported by the Scientific Research Fund of Sichuan Provincial Education Department(12ZA098), Artificial Intelligence of Key Laboratory of Sichuan Province(2012RYY04), and Fundamental Research Funds for the Central Universities.

\section{References}

[1] B. Schweizer and J. Smital, Measure of chaos and a spectral decomposition 6of dynamical systems of interval. Trans. Amer. Math. Soc, (344)1994, 737-754. 
[2] C. Tian and G. Chen, Chaos in the sence of Li-Yorke in coupled map lattices. Physica A, (376)2007, 246-252.

[3] F. J. Solis, L. Joder and B. Chen, Chaos in the one-dimensional wave equation. Applied Mathematics Letter. (18)2005, 85-90.

[4] F. Khellat, A. Ghaderi and N. Vasegh, Li-Yorke chaos and synchronous chaos in a globally nonlocal coupled map lattice. Chaos, Solitons and Fractals. (44)2011, 934-939.

[5] J. Auslander and J. A. Yorke, Interval maps, factors of maps and chaos. Tohoku math. J. (32)1980, 177-188.

[6] J. Banks, J. Brooks, G. Cairns, G. Davis and P. Stacey, On Devaney definition of chaos. Amer. math. Monthly, (99)1992, 332-334.

[7] L. Snoha, dense chaos. Comment. Math. Univ. Carolin. (33)1992, 747-752.

[8] L. Wang, G. Huang and S. Huan, Distributional chaos in a sequence. Nonlinear Analysis. (67)2007, 2131-2136.

[9] P. Oprocha, Relations between distributional and Devaney chaos. Chaos. (16)2006, 033112-033112-5.

[10] R. Hric and M. Malek, Omega limit sets and distributional chaos on graphs. Topology and its Applications. (153)2006, 2469-2475.

[11] R. L. Devaney, An introduction to chaotic dynamical system. Addison Wesley, 1989.

[12] T. Li and J. Yorke, Period 3 implies chaos. Amer. math. Monthly, (82)1975, 985-992.

\section{Received: May 23, 2013}

